may be exacerbated in pregnancy, suggesting a hormone or growth factor important in tumor formation. The inherited gene for NF2 is on the long arm of chromosone 22. (Martuza RL, Eldridge R. Neurofibromatosis 2 (Bilateral acoustic neurofibromatosis). N Engl J Med March 17 1988;318:684-8).

COMment. The British Neurofibromatosis Association -- LINK, and two voluntary support organizations in the US -- The National Neurofibromatosis Foundation and the Acoustic Neuroma Association, organize medical symposia and provide valuable assistance to patients and their families. Newer diagnostic methods including brain-stem auditory evoked potentials, CT and MRI and routine eye examination for subcapsular cataracts should afford earlier recognition.

Unidentified bright objects (UBO's), areas of increased signal on $\mathrm{Tl}$ or T2 weighted MRI, with concurrent negative contrast-enhanced CT are reported in 28 children with neurofibromatosis 1 and may possibly represent heterotopias. They were not correlated with the presence of learning disorders or retardation, and their significance is unclear. (Rubinstein $\mathrm{AE}$ et al. and Dunn DW, Roos KL. Neurology March 1988;38 (Suppl 1);282). I have a patient, a boy aged 12 yrs, with migraine headaches, macrocephaly, and cafe au lait patches, whose MRI shows UBO's. Although considered benign, such patients deserve careful long-term follow-up.

\title{
INPANTILE SEIZURES
}

\section{NEONATAL SEIZURES}

The clinical patterns, etiologies and times of onset of seizures were determined in a retrospective study of 150 newborns with seizures evaluated between 1982-87 in the neonatal intensive care unit at Washington University School of Medicine, Children's Hospital, St. Louis, MO. Subtle seizures in 97 (65\%) infants were characterized by eye movements, sucking, lip-smacking, chewing, tonic posturing, pedaling or rowing movements, and/or apneic spells. other seizure patterns were multifocal clonic (54\%), focal clonic, generalized tonic and myoclonic. Of $78 \%$ patients with at least one EEG, only $11 \%$ had ictal tracings. Hypoxicischemic encephalopathy (HIE) in $65 \%$ of the total was the most common cause of seizures in both preterm and term infants and $90 \%$ occurred in the first two days. Intracranial hemorrhage accounted for $10 \%$ of seizures. Other less common causes were metabolic, developmental defect, infection and drug withdrawal. In those with developmental defects, the onset of seizures was after 7 days of age. (Calciolari G, Perlman JM, Volpe JJ. Seizures in the neonatal intensive care unit of the 1980's. Clinical Pediatrics March 1988; 27:119-23).

COMranr. The authors admit that the absence of simultaneous video-EEG monitoring may lead to misinterpretation of the nature of some clinical phenomena regarded as seizures. Subtle seizures reported in more than half of the patients and associated with HIE have an inconsistent or no relationship to EEG seizure discharges (Mizrahi EM, Kellaway P. Neurology 1987;37:1837), and some authorities advise against anticonvulsant therapy unlesS EEG epileptiform confirmation is obtained (Ped Neur Briefs $1987 ; 1: 47-8)$. Self-resolution of "seizures" cannot be ruled out in a proportion of those apparently responding to anticonvulsant drugs, a therapy not without potential toxicity. 